\title{
Intensification of Mixing Processes with Complex Fluids
}

\author{
Naoto OhmurA ${ }^{1,4,5}$, Hayato Masuda ${ }^{2,4}$ and Steven WANG ${ }^{3,4}$ \\ ${ }^{1}$ Department of Chemical Science and Engineering, Kobe University, 1-1 Rokkodai, Nada-ku, Kobe-shi, \\ Hyogo 657-8501, Japan \\ ${ }^{2}$ School of Food and Nutritional Science, University of Shizuoka, 52-1 Yada, Suruga, Shizuoka-shi, \\ Shizuoka 422-8526, Japan \\ ${ }^{3}$ Department of Chemical Engineering and Advanced Materials, Newcastle University, \\ Newcastle upon Tyne, U.K. \\ ${ }^{4}$ Complex Fluid and Thermal Engineering Research Center (COFTEC), Kobe University, 1-1 Rokkodai, \\ Nada, Kobe-shi, Hyogo 657-8501, Japan \\ ${ }^{5}$ Research Unit for Future Creation \& Innovation "Creative Dojo," Kobe University, 1-1 Rokkodai, Nada, \\ Kobe-shi, Hyogo 657-8501, Japan
}

Keywords: Mixing, Process Intensification, Complex Fluids, Non-Newtonian Fluids, Multiphase Flow

The present paper provides an overview of the intensification of mixing processes with complex fluids. First, mixing processes of rheologically complex fluids and their design methodology are discussed. An example of process intensification of mixing with rheologically complex fluids using a Couette-Taylor flow reactor is introduced. Second, the intensification of the mixing of a suspension with high concentration solids and a novel approach for solid-liquid separation using a stirred vessel are discussed. Finally, a perspective of the intensification of mixing processes with complex fluids is summarized.

\section{Introduction}

Although there are several definitions of process intensification, the common point among these definitions is that process intensification gives large benefits to stakeholders who are asserting influence over processes by a quantum leap in their performance. In this sense, intensification of mixing processes also should aim to dramatically improve mixing by controlling fluid flow. As Reay et al. (2008) pointed out, however, although mixing is a frequently used unit operation, it seems to be one of the less exciting areas of process engineering. In fact, when we take a look at the 16th century technology for retrieving gold from ore drawn by Agricola and De Re Metallica Libri (1556), the mixing technology using stirred vessels does not seem to have progressed much over the last four centuries (Stankiewicz and Moulijn, 2000). However, this does not mean that mixing technologies do not play an important role in process intensification. On the contrary, many processes cannot maintain their high performance without effective mixing, because mixing is almost always linked to other rate processes, i.e. chemical reactions and heat, mass and momentum transfers. According to Reay et al. (2008), a survey conducted by a mixing consultant revealed that attention to the mixing process typically yielded increases in plant productivity of $10-20 \%$, in some mixing in cases reaching

Received on May 8, 2017; accepted on July 3, 2017

DOI: $10.1252 /$ jcej.17we149

Correspondence concerning this article should be addressed to $\mathrm{N}$. Ohmura (E-mail address: ohmura@kobe-u.ac.jp).

Presented at the 5th Asian Conference on Mixing (ACOM), Yamagata, August 29-September 1, 2016
$40 \%$. In this sense, mixing problems are not only in mixing devices, but also in many chemical devices such as distillation columns, etc.

Interaction between molecules or phases significantly influence the fluid flow. There are a wide variety of industries dealing with fluids having such interactions that cannot be neglected. These fluids are called "complex fluids" (Masuda, 2016). Polymeric fluids and multiphase fluids are typical examples of complex fluids. In many industries such as food, mineral, paper, plastic and rubber industries, mixing has to deal with the complex fluids. Development, design and operation of a chemical process dealing with such complex fluids are quite difficult and there is a wide gap between theory and practice. In order to fill the gap, engineers frequently have to use their know-how based individual approaches. As previously mentioned, the mixing technology using stirred vessels does not seem to have progressed much over the last four centuries. In other words, however, an abundant amount of knowledge based on the engineers' experience has been accumulated for over 400 years. This might be one of the reasons why many industries cannot drastically change from all the existing chemical processes to novel ones. This is particularly the case with many established foods, commodity chemicals and mineral products (Wu et al., 2007). In order to comprehensively understand intensification of mixing processes, how to deal with these complex fluids should be discussed inevitably.

The present paper, therefore, overviews and discusses intensification of mixing processes with complex fluids by taking examples of rheologically complex fluids in food industries and multiphase complex fluids in mineral industries. 


\section{Mixing of Rheologically Complex Fluids}

\subsection{Introduction of mixing processes with rheologically complex fluids}

Rheologically complex fluids frequently appear in food industries. Roughly speaking, these fluids are classified into shear-thickening, shear-thinning, and viscoelastic fluids. Theses fluids sometimes have a yield stress. Shear-thinning fluids are perhaps most widely encountered type of nonNewtonian fluid in engineering practice. Due to the shearthinning property, the viscosity of fluid spatially changes with the spatial distribution of temperature in a reactor or a tank. Figure 1 shows the viscosity distribution in a vessel obtained by numerical simulation.

This viscosity distribution complicates the fluid flow compared with a Newtonian fluid system. For example, Fontaine et al. (2013) experimentally showed that, under the laminar condition, segregated zones in the tank with a Maxblend impeller were observed in a highly shear-thinning fluid system. Furthermore, according to Venneker et al. (2010), in the turbulent regime, all velocity components in a stirred vessel with a Rushton turbine are affected by the shear-thinning property of the fluids. Yield stress behavior is also a common property in food industries. It should be noted that the concept of yield stress is often discussed from the viewpoint of rheology (Barnes, 1999). In a stirred vessel, a yield stress fluid is mobile around the impeller where the shear stress is high, while the same fluid is stagnant away from the impeller where shear stress is low (Arratia et al., 2005, 2006). These mobile regions are called "caverns" (Wichterle and Wein, 1981). Many researchers have investigated the cavern size and shape including fluid dynamics in a tank (Solomon et al., 1981; Elson et al., 1986; Hirata et al., 1994; Hirata and Aoshima, 1996; Curran et al., 2000; Doucet et al., 2005). Although the models established in their studies are able to predict the cavern size under a range of operating conditions, the formation of the cavern itself is not avoidable unless abundant energy is injected to make the fluid circulate in the whole region. Furthermore, shear-thinning fluids having a yield stress is often encountered in food industries. In such a fluid system, there are many dead zones in a traditional continuous stirred tank reactor (Saeed and Ein-Mozaffari, 2008; Patel et al., 2011). On the basis of these problems, Niederkorn and Ottino (1994) state that the current practice of designing industrial mixers for nonNewtonian fluids is more art than science. Thus, from the basic perspective, a strategy of the design of a mixing device for non-Newtonian fluids should be discussed towards process intensification. In this section, first, the fundamental problem for the design of non-Newtonian fluid processes is discussed, and subsequently, examples of intensification of mixing process with rheologically complex fluids is reviewed.

\subsection{Design of process with rheologically complex fluid}

Before considering the intensification of a process with rheologically complex fluids, we need to pay attention to a

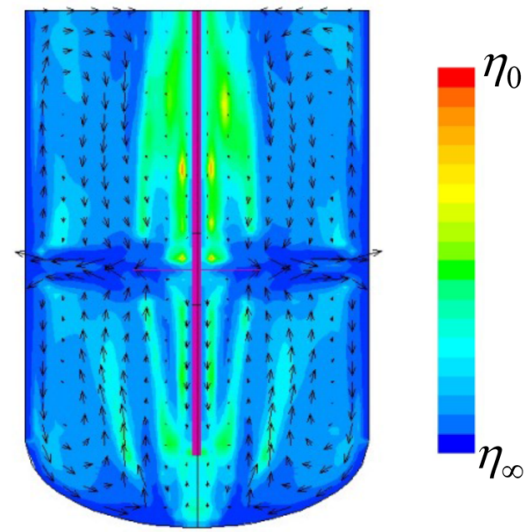

Fig. 1 Viscosity distribution and velocity vectors in a vessel with Rushton turbine; fluid is assumed to be a shear-thinning fluid; Carreau model was used as the rheological model; Reynolds number $(R e)$ was set at $R e=52$

fundamental problem on dealing with these fluids. In such fluid systems, the concept of the definition of the nondimensional number which is the basis for the design and operation of process is not well established yet. Fluid flows are governed by the Navier-Stokes equations. When the Navier-Stokes equations are expressed in a non-dimensional form, two non-dimensional parameters, Re (Reynolds number) and $\mathrm{Fr}$ (Froude number), appear in the forms of $1 / R e$ and $1 / F r$. It should be noted that, for a forced convection system, $R e$ is the only flow parameter. However, the definition of $R e$ is quite difficult because the viscosity spatially changes due to non-Newtonian property. As Ohta et al. $(2003,2005,2012)$ pointed out, from the practical viewpoint, the construction of effective $R e, R e_{\text {eff }}$, for the system based on the effective viscosity, $\eta_{\text {eff }}$ is required for the design of non-Newtonian fluid process.

In order to define $R e_{\text {effi, }}$ how to appropriately estimate the effective viscosity, $\eta_{\text {eff }}$, is the key subject. This is synonymous with how to estimate the effective shear-rate, $\dot{\gamma}_{\text {effi }}$ Usually, the value of $\dot{\gamma}_{\text {eff }}$ in a stirred tank is estimated by the Metzner-Otto method. Metzner and Otto (1957) suggested that the average shear-rate in the general region of the impeller could be taken as being directly proportional only to the speed of rotation of the impeller. Thus, they expressed the average shear-rate as Eq. (1).

$$
\dot{\gamma}_{\mathrm{av}}=K_{\mathrm{s}} \cdot N
$$

Here, $K_{\mathrm{s}}$ is the proportional constant which varies according to the impeller type and $N$ is the speed of rotation of the impeller. Although the Metzner-Otto's method is convenient due to its simplicity, their concept takes into consideration only the information around the vicinity of the impeller. Figure 2 shows the shear-rate distribution obtained from numerical simulation at $\dot{\gamma}_{\mathrm{av}}=200 \mathrm{~s}^{-1}$ for a Rushton turbine impeller. As clearly shown in Figure 2, the strong shear is locally added to the fluid around the vicinity of the impeller, and the shear-rate in large areas of the vessel is lower than the average shear-rate. Kaminoyama et al. (2011) proposed 

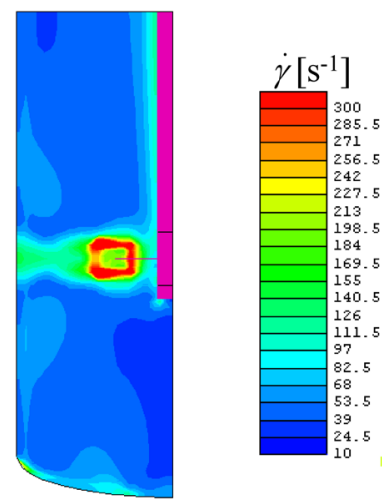

Fig. 2 Shear-rate distribution in the vessel with Rushton turbine at $\dot{\gamma}_{\mathrm{av}}=200 \mathrm{~s}^{-1}$

a new method for determining the effective viscosity of a shear-thinning fluid in a stirred vessel. Their method is that $\eta_{\text {eff, }}$ which is the average of the local viscosity distribution in the vessel using a weight of a dissipation function, is determined from the results of the numerical simulations alone. They confirmed that the reliability of this method was ascertained since the conservation of flow similarity was better than that achieved with the conventional Metzner-Otto method, when scaling up the vessels under the condition that the $R e_{\text {eff }}$ calculated with this method is constant. In shear-thinning fluid systems, this method for the estimation of effective viscosity has been applied for other flow systems and verified, e.g., Taylor-Couette flow (Masuda et al., 2015, 2017b) and Rayleigh-Bénard convection (Parmentier 1978; Jenny et al., 2015).

Some researchers have reported that $K_{s}$ depends on not only an impeller type, but also rheological properties of a fluid (e.g., Rieger and Novak, 1973; Šesták et al., 1986; Carreau et al., 1992; Tanguy et al., 1996; Anne-Archard et al., 2006). Also, Masuda et al. (2017a) indicated that some revisions for the Metzner-Otto method are necessary when their concept is applied to a particle aggregation process using a stirred vessel for the estimation of $\dot{\gamma}_{\text {eff. }}$. In the future, revising the Metzner-Otto concept will be necessary including the application to viscoelastic fluid processes.

\subsection{Intensification of mixing processes with rheologically complex fluids}

In order to intensify the mixing processes with rheologically complex fluids, a novel mixing device has been proposed. For example, Hubacz and Buczynska $(2011,2013)$ and Masuda et al. $(2013,2017 \mathrm{c})$ applied the Taylor-Couette flow reactor to the continuous starch hydrolysis process. At high starch content $\left(C_{0}\right)$, gelatinized starch becomes highly viscous shear-thinning fluids. Thus, it is difficult to mix the gelatinized starch with enzyme if a conventional stirred vessel is used. Figure 3 shows the dependency of yield of reducing sugar on $R e_{\text {eff }}$ at $C_{0}=50-300 \mathrm{~g} / \mathrm{L}$. As shown in Figure 3 , starch at high contents in the slurry was hydrolyzed effectively and continuously in the Taylor vortex flow region where $R e_{\text {eff }}$ is higher than $R e_{\text {cr }}$. Another example is the heat

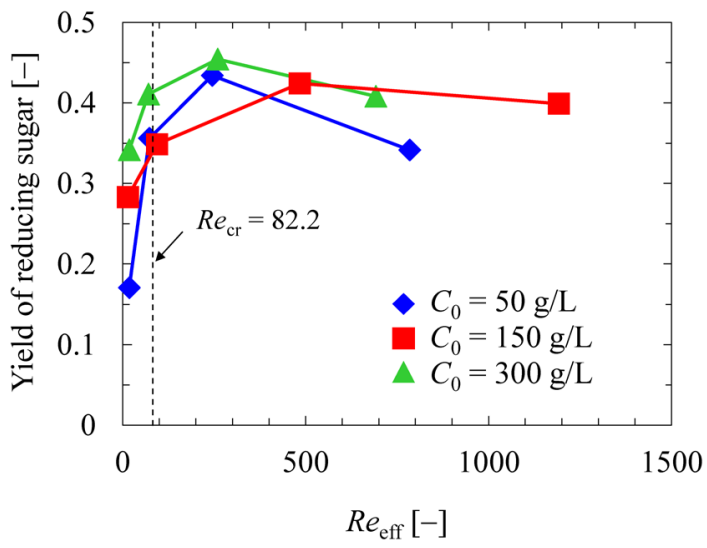

Fig. 3 Dependency of yield of reducing sugar on $R e_{\text {eff: }}$ the dashed line shows $R e_{\text {cr }}$ where Taylor vortices begin to form

sterilization of foods having the shear-thinning property using the Dean vortex by Kelder et al. (2002). They numerically showed that increasing the strength of swirl motion due to the Dean vortex promotes heat transfer, and thus the lethality of spores in foods was increased. The unique motion of fluid like Taylor-Couette flow or Dean vortex flow should be positively utilized as mixing devices.

In the conventional stirred vessel, the steady mixing by the impeller would face a limitation for dealing with rhelogically complex fluids because the formation of an unmixed region, like caverns, is not avoidable under the steady flow field. Chaotic mixing under an unsteady flow field is considered to be an effective breakthrough because the localization of an unmixed region would be avoidable. Although the chaotic mixing with non-Newtonian fluids has been investigated by several researchers, there is very little information about it compared with Newtonian fluid systems. Furthermore, most of these studies have been limited to two-dimensional flow until now (Leong and Ottino, 1990; Niederkorn and Ottino, 1993, 1994; Arratia et al., 2005, 2006). Although Anderson et al. (2000) investigated mixing in three-dimensional time-periodic cavity flow, the geometry is a simple rectangular. In a stirred vessel, the unsteady flow field is generated by the simple means like an unsteady rotational speed of the impeller (Yao et al., 1998), or a reciprocating motion of the impeller (Komoda et al., 2000). Studies of chaotic mixing in the stirred vessel with Newtonian fluid processes are reviewed by Takahashi (2007) in detail. The extension of the knowledge obtained until now to nonNewtonian fluid processes would be necessary for the intensification of mixing process.

\section{Mixing of Multiphase Fluids}

\subsection{Mixing of suspension with high concentration solids}

In the context of the mineral industry, a great number of hydro-metallurgical processes are adopted to obtain high purity metals from their raw ores. In those hydro-refining processes, slurry-holding tanks, leaching tanks, extraction units and stirred reactors are typically used to homogenize 
the reactive/non-reactive materials. In these tanks, high solid loading $(>0.20 \mathrm{v} / \mathrm{v})$ and complex slurry rheology are often encountered, as a large volume of mineral particles are involved in the hydro-metallurgical processes. This suggests that high operation costs are usually incurred, because excessive 'mixing' power is required for suspending or homogenizing the high concentration particles in the slurry. In order to maintain the given goals, process intensification appears to be one attractive strategy to notably minimize unit volume while keeping the productivity in the mineral refineries. The ultimate goal of process intensification was proposed to intensify the mixing units that deal with ultra-high concentration reactive and/or inert particles in complex fluids (Wang et al., 2012a, 2012b, 2016a; Wu et al., 2016).

Ultra-high (fine) solids loadings tend to modify slurry viscosity and/or rheology, and recent experimental and theoretical analysis have showed that homogenization of high concentration slurries in the stirred reactors is rather difficult (Wang et al., 2012a, 2012b, 2016a). The challenges are linked to the formation of stagnant regions (e.g., cavern) and isolate mixing regions (IMRs), settling of heavy particles and flotation of light particles (Wang et al., 2013; Wu et al., 2016). These issues lead to extremely poor heat and mass transfer owing to a low degree of homogeneity. In a recent work, Wang et al. (2016a) showed that cloud height can be used as the criteria to measure the slurry homogeneity. In their numerical and experimental studies, they showed that the removal of vertical baffles is a viable method to intensify the mixing process as power consumption can be massively reduced. This approach is novel and effective considering the fact that complete suspension of fine particles can be achieved, the surface vortex can be damped by the high concentration particles, and impeller consumption can be also reduced massively.

Wu et al. (2016) also found that fine particles could be suspended in the upper region of the baffled iron slurry tank. This phenomenon is illustrated in Figure 4. They found that, in a conventional baffled tank, the status of floating (fine) particles could be unchanged even when the system is operated at a high very speed. This appears to be problematic because fine additives are usually blended with other materials in the industrial iron ore mixing process. In the most severe operation, a massive amount of floating fine particles could result in waste of valuable additives, consequently leading to adverse process economics. In this case, an increase in impeller clearance is not feasible because the impeller is also responsible for suspending heavy particles in these multiphase systems.

In consideration of process intensification, the removal of baffles seems to be a reliable approach based on the laboratory and industrial practices. As shown in Figure 5, in a pilot scale tank, at the same power input, the unbaffled tank appears to be superior in handling the fine particles in the complex multiphase system. In Figure 5b, in contrast to the baffled system, it shows that the thickness of stagnant fine particles is almost negligible, implying that a much higher degree of homogeneity is obtained.

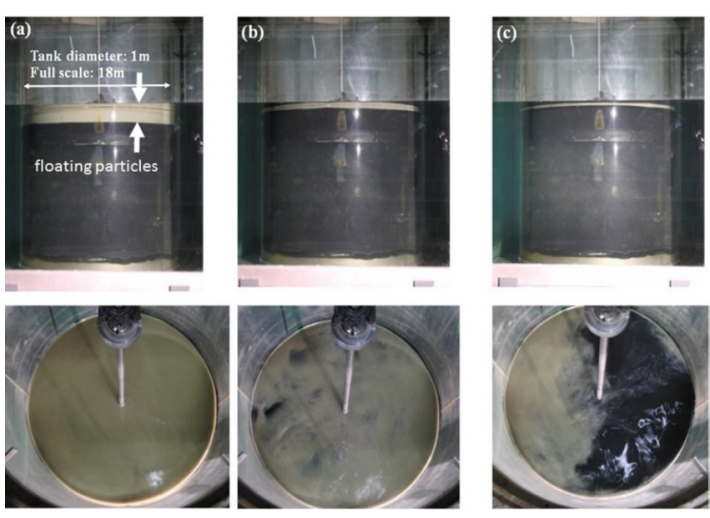

Fig. 4 Dispersion of low-density silica particles in a pilot-scale rotating tank (a) Speed $=195 \mathrm{rpm}$; (b) Speed $=235 \mathrm{rpm}$; (c) Speed $=275 \mathrm{rpm}$; in the system, magnetite solids: $0.25 \mathrm{v} / \mathrm{v}$, water: $0.63 \mathrm{w} / \mathrm{w}$, silica particles: $0.016 \mathrm{v} / \mathrm{v}$; tank diameter: $1 \mathrm{~m}$. (Wu et al., 2016)
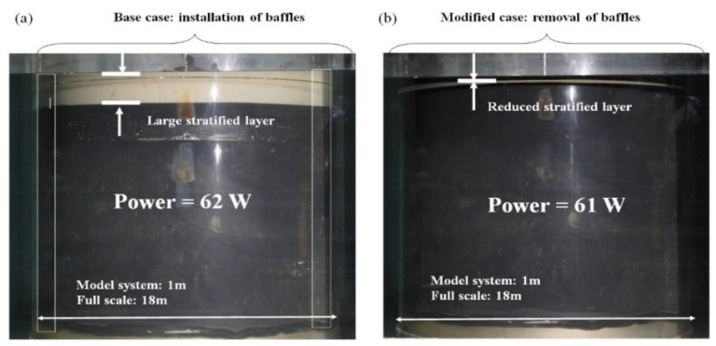

Fig. 5 Impact of removal of baffles on dispersion of particles in a pilot-scale stirred vessel (a) with baffles; (b) without baffles. Tank diameter: $1 \mathrm{~m}$. (Wu et al., 2016)

This finding is linked to the fact that, in a baffled tank, fluid velocities are very weak in the upper region of the system, and the removal of baffles homogenizes the fluid velocities through the system.

\subsection{A novel approach for solid-liquid separation using a stirred vessel}

It is very well known that the dynamics of finite-sized particles could be strikingly different from that of passive particles. In our earlier discovery, this has been proved again in a conventional stirred tank in that, even in laminar flows $(R e<100)$, non-passive particles can tend to move independently from the underlying fluid streamlines, and subsequently move into the so-called 'isolated mixing regions.' The physics have been discussed in Wang et al. (2014), and is illustrated in Figure 6.

In the context of biotechnological processes, we usually encounter some multiphase systems where difficultto-separate suspensions are involved. Difficult-to-separate suspensions refer to slurry systems in which there is some combination of a small density difference between solid and liquid, high viscosity, and small-sized particles. It should be noted that no effective technologies are actually available to handle these suspensions. Wang et al. (2014) proposed that the unexpected trapping physics (as shown in Figure 6) can be manipulated to effectively and efficiently separate 


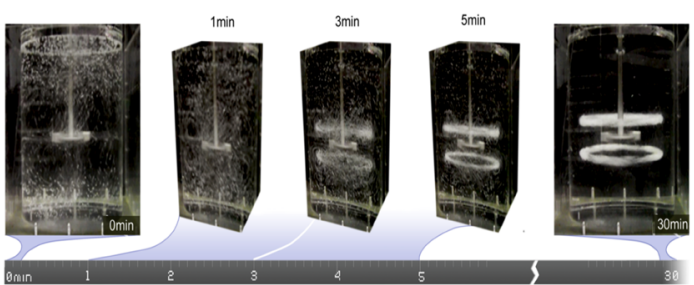

Fig. 6 Trapping of non-passive particles in a laminar multiphase system. $R e \sim 100$

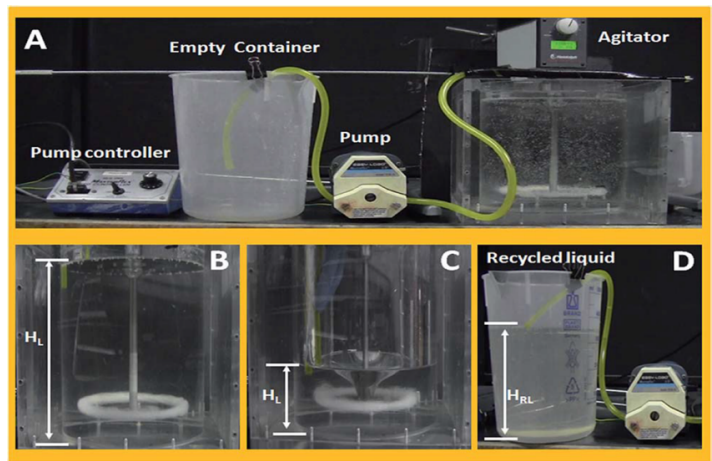

Fig. 7 Solid-liquid separation in a highly viscous multiphase system

difficult-to-separate suspensions. The separation of relatively small particles (few hundred microns in diameter) from the highly viscous liquid $(\sim 100 \mathrm{~Pa} \cdot \mathrm{s})$ makes it possible to achieve the ultimate goal of process intensification, which tends to minimize the capital/operation inputs to achieve the same outcome. The demonstration is shown in Figure 7. In this setup, the relatively simple setup consisting of a small unbaffled tank, an overhead stirrer and a liquid pump can allow removal of $\sim 75 \%$ purified liquid with minimal energy input (Wang et al., 2014, 2016b).

\section{Conclusions}

Process intensification of mixing with complex fluids is still one of the most challenging issues in the chemical engineering field. Because, as mentioned in the introduction, many industries such as food, mineral, paper, plastic and rubber industries, have to deal with the complex fluids in their mixing processes.

When we overview the mixing processes discussed in the previous sections, we can notice that swirl and circulating (vortex) motions are crucially important building blocks of mixing fields. As for vortex motion, utilizing appropriate vortex motions can significantly enhance the energy efficiency of unit operations and make chemical processes more compact and safer. Some process intensification methods are, therefore, closely related to vortex dynamics (Wang and Ohmura, 2017). How to incorporate the swirl and vortex dynamics into mixing fields is a key issue for the process intensification of mixing with complex fluids.

As for rheological complexity, the rheological properties of fluids often change not only spatially, but also temporally.
How to overcome this temporal-spatial inhomogeneity is, therefore, another key issue for process intensification. Conversion from a batch to a continuous process using a Couette-Taylor reactor discussed in this paper might provide insight into this issue. Theoretically speaking, a plug-flow type reactor such as a Couette-Taylor reactor and an oscillatory baffled reactor (Mackley and $\mathrm{Ni}, 1991$ ) can convert temporal change to spatial. If such a plug-flow type reactor can be divided into several well-controlled mixing compartments in the bulk flow direction, this problem on temporal-spatial inhomogeneity can be solved.

Toward the intensification of mixing processes with complex fluids, computational fluid dynamics (CFD) is one of the powerful tools as it can give valuable information on mixing fields. In order to effectively utilize CFD, precise models and their validation against a large amount of experimental data are required. In addition, as previously mentioned, abundant knowledge of mixing technologies based on the experience of engineers has been accumulated for over 400 years. In these senses, data scientific approaches dealing with big data might be necessary to pave the way for the development of innovative mixing devices in the very near future.

\section{Acknowledgement}

This research was financially supported by a Grant-in-Aid for Challenging Exploratory Research (No. 15K14205) from Japan Society for the Promotion of Science (JSPS).

\section{Nomenclature}

$C_{0} \quad=$ initial content of starch $\quad[\mathrm{g} / \mathrm{L}]$

$\mathrm{Fr} \quad=$ Froude number $[-]$

$K_{\mathrm{s}} \quad=$ Metzner-Otto constant $\quad[-]$

$L=$ representative length $[\mathrm{m}]$

$N \quad=$ rotational speed of the impeller $\left[\mathrm{s}^{-1}\right]$

Re $\quad=$ Reynolds member $\quad[-]$

$R e_{\mathrm{cr}} \quad=$ critical Reynolds number $\quad[-]$

$R e_{\text {eff }} \quad=$ effective Reynolds number $\quad[-]$

$\dot{\gamma}_{\mathrm{av}}=$ average shear-rate $\left[\mathrm{s}^{-1}\right]$

$\dot{\gamma}_{\text {eff }}=$ effective shear-rate $\left[\mathrm{s}^{-1}\right]$

$\eta_{\text {eff }}=$ effective viscosity $\quad[\mathrm{Pa} \cdot \mathrm{s}]$

\section{Literature Cited}

Agricola, G. and X. De Re Metallica Libri; II, Froben \& Episopius, Basel, Switzerland (1556)

Anderson, P. A., O. S. Galaktionov, G. W. M. Peters, F. N. van de Vosse and H. E. H. Meijer; "Mixing of Non-Newtonian Fluids in TimePeriodic Cavity Flows," J. Non-Newt. Fluid Mech., 93, 265-286 (2000)

Anne-Archard, D., M. Marouche and H. C. Boisson; "Hydrodynamic and Metzner-Otto Correlation in Stirred Vessels for Yield Stress Fluids," Chem. Eng. J., 125, 15-24 (2006)

Arratia, P. E., G. A. Voth and J. P. Gollub; "Stretching and Mixing of Non-Newtonian Fluids in Time-Periodic Flows," Phys. Fluids, 17, 053102 (2005)

Arratia, P. E., J. Kukura, J. Lacombe and F. J. Muzzio; "Mixing of Shear- 
Thinning Fluids with Yield Stress in Stirred Tanks," AIChE J., 52, 2310-2322 (2006)

Barnes, H. A.; "The Yield Stress-A Review or ' $\pi \alpha v \tau \alpha \rho \varepsilon$ '-Everything Flows?" J. Non-Newt. Fluid Mech., 81, 133-178 (1999)

Carreau, P. J., J. Paris and P. Guérin; "Mixing of Newtonian and NonNewtonian Liquids: Screw Agitator and Draft Coil System," Can. J. Chem. Eng., 70, 1071-1082 (1992)

Curran, S. J., R. E. Hayes, A. Afacan, M. C. Williams and P. A. Tanguy; "Experimental Mixing Study of a Yield Stress Fluid in a Laminar Stirred Tank," Ind. Eng. Chem. Res., 39, 195-202 (2000)

Doucet, L., G. Ascanio and P. A. Tanguy; "Hydrodynamics Characterization of Rotor-Stator Mixer with Viscous Fluids," Chem. Eng. Res. Des., 83, 1186-1195 (2005)

Elson, T. P., D. J. Cheesman and A. W. Nienow; "X-Ray Studies of Cavern Sizes and Mixing Performance with Fluids Possessing a Yield Stress," Chem. Eng. Sci., 41, 2555-2562 (1986)

Fontaine, A., Y. Guntzburger, F. Bertrand, L. Fradette and M.-C. Heuzey; "Experimental Investigation of the Flow Dynamics of Rheologically Complex Fluids in a Maxblend Impeller System Using PIV," Chem. Eng. Res. Des., 91, 7-17 (2013)

Hirata, Y. and Y. Aoshima; "Formation and Growth of Cavern in Yield Stress Fluid Agitated Under Baffled and Non Baffled Conditions," Chem. Eng. Res. Des., 74, 438-444 (1996)

Hirata, Y., A. W. Nienow and I. P. T. Moore; "Estimation of Cavern Sizes in a Shear-Thinning Plastic Fluid Agitated by a Rushton Turbine Based on LDA Measurements," J. Chem. Eng. Japan, 27, 235-237 (1994)

Hubacz, R. and M. Buczynska; "Starch Gelatinization in Couette-Taylor Flow Apparatus," Chem. Process Eng., 32, 267-279 (2011)

Hubacz, R., N. Ohmura and E. Dluska; "Intensification of Starch Processing Using Apparatus with Couette-Taylor Flow," J. Food Process Eng., 36, 774-785 (2013)

Jenny, M., E. Plaut and A. Briard; "Numerical Study of Subcritical Rayleigh-Bénard Convection Rolls in Strongly Shear-Thinning Carreau Fluids," J. Non-Newt. Fluid Mech., 219, 19-34 (2015)

Kaminoyama, M., K. Nishi, R. Misumi and F. Otani; "A Method for Determining the Representative Apparent Viscosity of Highly Viscous Pseudoplastic Liquids in a Stirred Vessel by Numerical Simulation," J. Chem. Eng. Japan, 44, 868-875 (2011)

Kelder, J. D. H., K. J. Ptasinski and P. J. A. M. Kerkhof; "Power-Law Foods in Continuous Coiled Sterilisers," Chem. Eng. Sci., 57, 46054615 (2002)

Komoda, Y., Y. Inoue and Y. Hirata; "Mixing Performance by Reciprocating Disk in Cylindrical Vessel," J. Chem. Eng. Japan, 33, 879-885 (2000)

Leong, C. W. and J. M. Ottino; "Increase in Regularity by Polymer Addition during Chaotic Mixing in Two-Dimensional Flows," Phys. Rev. Lett., 64, 874-877 (1990)

Mackley, M. R. and X. Ni; "Mixing and Dispersion in a Baffled Tube for Steady Laminar and Pulsatile Flow," Chem. Eng. Sci., 46, 31393151 (1991)

Masuda, H.; "Process Intensification of Taylor-Couette Flow Reactor with Complex Fluids," Ph. D. dissertation, Kobe University, Japan (2016)

Masuda, H., T. Horie, R. Hubacz and N. Ohmura; "Process Intensification of Continuous Starch Hydrolysis with a Couette-Taylor Flow Reactor," Chem. Eng. Res. Des., 91, 2259-2264 (2013)

Masuda, H., T. Horie, R. Hubacz, M. Ohta and N. Ohmura; "Numerical Analysis of the Flow of Fluids with Complex Rheological Properties in a Couette-Taylor Flow Reactor," Theor. Appl. Mech. Japan, 63, 25-32 (2015)

Masuda, H., K. Tsuda, K. Matsui, Y. Komoda and N. Ohmura; "Effect of
Shear Rate Distribution on Particle Aggregation in a Stirred Vessel," Chem. Eng. Technol., 40, 493-497 (2017a)

Masuda, H., T. Horie, R. Hubacz, M. Ohta and N. Ohmura; "Prediction of Onset of Taylor-Couette Instability for Shear-Thinning Fluids," Rheol. Acta, 56, 73-84 (2017b)

Masuda, H., T. Horie, R. Hubacz, N. Ohmura and M. Shimoyamada; "Process Development of Starch Hydrolysis Using Mixing Characteristics of Taylor Vortices," Biosci. Biotechnol. Biochem., 81, $755-761(2017 \mathrm{c})$

Metzner, A. B. and R. E. Otto; "Agitation of Non-Newtonian Fluids," AIChE J., 3, 3-10 (1957)

Niederkorn, T. C. and J. M. Ottino; "Mixing of Viscoelastic Fluid in a Time-Periodic Flow," J. Fluid Mech., 256, 243-268 (1993)

Niederkorn, T. C. and J. M. Ottino; "Chaotic Mixing of Shear-Thinning Fluids," AIChE J., 40, 1782-1793 (1994)

Ohta, M., E. Iwasaki, E. Obata and Y. Yoshida; "A Numerical Study of the Motion of a Spherical Drop Rising in Shear-Thinning Fluid Systems," J. Non-Newt. Fluid Mech., 116, 95-111 (2003)

Ohta, M., E. Iwasaki, E. Obata and Y. Yoshida; "Dynamic Processes in a Deformed Drop Rising Through Shear-Thinning Fluids," J. NonNewt. Fluid Mech., 132, 100-107 (2005)

Ohta, M., S. Kimura, T. Furukawa, Y. Yoshida and M. Sussman; "Numerical Simulations of a Bubble Rising Through a Shear-Thickening Fluid," J. Chem. Eng. Japan, 45, 713-720 (2012)

Parmentier, E. M.; "A Study of Thermal Convection in Non-Newtonian Fluids," J. Fluid Mech., 84, 1-11 (1978)

Patel, V. R., F. Ein-Mozaffari and S. R. Upreti; "Effect of Time Delays in Characterizing the Continuous Mixing of Non-Newtonian Fluids in Stirred-Tank Reactors," Chem. Eng. Res. Des., 89, 1919-1928 (2011)

Reay, D., C. Ramshow and A. Harvey; Process Intensification, 1st ed., Butterworth-Heinemann Oxford, U.K. (2008)

Rieger, F. and V. Novak; "Power Consumption of Agitators in Highly Viscous Non-Newtonian Liquids," Trans. Ins. Chem. Eng., 51, 105-111 (1973)

Saeed, S. and F. Ein-Mozaffari; "Using Dynamic Tests to Study the Continuous Mixing of Xanthan Gum Solutions," J. Chem. Technol. Biotechnol., 83, 559-568 (2008)

Šesták, J., R. Žitný and M. Houška; "Anchor-Agitated Systems: Power Input Correlation for Pseudoplastic and Thixotropic Fluids in Equilibrium," AIChE J., 32, 155-158 (1986)

Solomon, J., T. P. Elson, A. W. Nienow and G. W. Pace; "Cavern Sizes in Agitated Fluids with a Yield Stress," Chem. Eng. Commun., 11, 143-164 (1981)

Stankiewicz, A. and J. A. Moulijn; "Process Intensification: Transforming Chemical Engineering," Chem. Eng. Prog., 96, 22-34 (2000)

Takahashi, K.; "Recent Advance in Chaotic Mixing in a Mixing Equipment," J. Chem. Eng. Japan, 40, 605-610 (2007)

Tanguy, P. A., F. Thibault and E. B. De La Fuente; "A New Investigation of the Metzner-Otto Concept for Anchor Mixing Impellers," Can. J. Chem. Eng., 74, 222-228 (1996)

Venneker, B. C. H., J. J. Derksen and H. E. A. Van den Akker; "Turbulent Flow of Shear-Thinning Liquids in Stirred Tanks-The Effects of Reynolds Number and Flow Index," Chem. Eng. Res. Des., 88, 827-843 (2010)

Wang, S., R. Parthasarathy, E. Bong, J. Wu and P. Slatter; "Suspension of Ultrahigh Concentration Solids in an Agitated Vessel," AIChE J., 58, 1291-1298 (2012a)

Wang, S., D. Boger and J. Wu; "Energy Efficient Solids Suspension in an Agitated Vessel-Water Slurry," Chem. Eng. Sci., 74, 233-243 (2012b)

Wang, S., J. Wu and N. Ohmura; "Inclined-Shaft Agitation for Improved 
Viscous Mixing," Ind. Eng. Chem. Res., 52, 11741-11751 (2013)

Wang, S., G. Metcalfe, R. Stewart, J. Wu, N. Ohmura, X. Feng and C. Yang; "Solid-Liquid Separation by Particle-Flow-Instability;" Energy Environ. Sci., 7, 3982-3988 (2014)

Wang, S., M. Jiang, S. Ibrahim, J. Wu, X. Feng, X. Duan, Z. Yang, C. Yang and N. Ohmura; "Optimized Stirred Reactor for Enhanced Particle Dispersion,” Chem. Eng. Technol., 39, 680-688 (2016a)

Wang, S., R. Stewart and G. Metcalfe; "Visualization of the Trapping of Inertial Particles in a Laminar Mixing Tank," Chem. Eng. Sci., 143, 99-104 (2016b)

Wang, S. and N. Ohmura; "Dynamical Particle Motions in Vortex Flows," in Vortex Dynamics and Optical Vortices, H. Perez-De-
Tejada eds., pp. 133-150, INTECH, Rijeka, Croatia (2017)

Wichterle, K. and O. Wein; "Threshold of Mixing of Non-Newtonian Fluids," Int. Chem. Eng., 21, 116-120 (1981)

Wu, J., L. J. Graham and M. N. Noui-Mehidi; "Intensification of Mixing," J. Chem. Eng. Japan, 40, 890-895 (2007)

Wu, J., S. Wang, B. Nguyen, D. Marjavaara and O. Eriksson; "Improved Mixing in a Magnetite Iron Ore Tank via Swirl Flow: Lab-Scale and Full-Scale Studies," Chem. Eng. Technol., 39, 505-514 (2016)

Yao, W. G., H. Sato, K. Takahashi and K. Koyama; "Mixing Performance Experiments in Impeller Stirred Tanks Subjected to Unsteady Rotational Speed," Chem. Eng. Sci., 53, 3031-3040 (1998) 